\title{
Introduction au dossier
}

La plage, territoire des corps

Vincent Coëffé, Emmanuel Jaurand et Benjamin Taunay

\section{CpenEdition}

\section{Journals}

Édition électronique

URL : http://journals.openedition.org/tourisme/135

DOI : 10.4000/tourisme.135

ISSN : 2492-7503

Éditeur

Éditions touristiques européennes

Édition imprimée

Date de publication : 1 juin 2014

Pagination : $32-41$

ISSN : 2109-5671

Référence électronique

Vincent Coëffé, Emmanuel Jaurand et Benjamin Taunay, «Introduction au dossier », Mondes du

Tourisme [En ligne], 9 | 2014, mis en ligne le 15 septembre 2015, consulté le 22 septembre 2020. URL :

http://journals.openedition.org/tourisme/135; DOI : https://doi.org/10.4000/tourisme.135

\section{$\theta \Theta \Theta \Theta$}

Mondes du tourisme est mis à disposition selon les termes de la licence Creative Commons Attribution - Pas d'Utilisation Commerciale - Pas de Modification 4.0 International. 


\section{INTRODUGTION AU DOSSIER}

\section{La plage, territoire des corps}

\section{Vincent Cö̈rfÉ \\ Maître de conférences en géographie Université d'Angers, UMR CNRS 6590 Espaces et Sociétés (ESO) [vincent.coeffe@univ-angers.fr]}

\section{Emmanuel Jaurand \\ Professeur de géographie Université d'Angers, UMR CNRS 6590 Espaces et Sociétés (ESO) [emmanuel.jaurand@univ-angers.fr]}

\section{Benjamin Taunay}

Maître de conférences en géographie Université d'Angers, UMR CNRS 6590 Espaces et Sociétés (ESO) [benjamin.taunay@univ-angers.fr]
64 es plages vont-elles disparaître?", se demandait en 2005 Roland Paskoff. Question qui peut paraître étrange alors que nous devons constater combien cet objet fait aujourd'hui partie de notre monde et qu'il constitue même une réalité suscitant un faisceau d'images qui parcourent tout le spectre des valeurs. Afin de comprendre cette apparente contradiction, nous n'oublierons toutefois pas de noter que cette question inquiète était celle d'un géographe physicien. Et ce n'est pas un hasard.

La plage est un terme dont le sens semble d'abord renvoyer à une réalité (géo)morphologique. La plage, en effet, est longtemps restée un objet monopolisé par les géographies dites physiques, notamment à travers la géomorphologie du littoral qui la considère comme "un secteur de côte où des sédiments, sables et galets sont accumulés par la mer" (Paskoff, 2005). Cette filiation avec les sciences naturelles pose de plus le problème du positionnement de la géographie parmi les sciences sociales, dont l'intégration à ce champ de disciplines a été retardée par les premiers choix des pères fondateurs de la géographie française, tel Paul Vidal de La Blache.

Cela dit, la plage reste discrète dans les sciences sociales en général. Ces blocages sont liés à des postures théoriques et épistémologiques, plus ou moins fortes selon les disciplines. Ainsi, en géographie, on citera d'abord la faible valeur accordée au thème des loisirs au regard de celle attachée aux activités productives (agricoles, industrielles), une configuration qui a aussi pendant longtemps affaibli la légitimité de l'objet touristique. Le privilège accordé à la sédentarité, ensuite, est presque rédhibitoire : en témoigne chez les géographes, par exemple, la célébration par l'École française (de géographie) de la région naturelle ou paysagère, largement assise sur 
des critères géologiques. L'ordre de la sédentarité a aggravé les effets précédemment décrits, en éloignant un peu plus les pratiques touristiques des préoccupations des sciences sociales. On peut également souligner la primauté de l'échelon régional ou national, notamment en géographie là encore, sur les analyses déployées aux niveaux géographiques les plus fins. Du coup, l'échelon microgéographique, et $a$ fortiori celui du corps, a été plus ou moins négligé, en dépit de la prise en compte des spatialités liées à la gestion des distances interpersonnelles chez certains anthropologues et sociologues (Hall, 1984 ; Goffman, 1973). Enfin, et de manière connexe au précédent blocage, les sciences sociales sont questionnées par le tabou du corps, singulièrement celui du corps dénudé, souvent associé dans l'imaginaire à la sexualité. D'ailleurs, cette dernière est encore assez peu investie par la recherche française en géographie ou par les études touristiques, même si des travaux récents viennent nuancer ce propos (Stock et Volvey, 2003 ; Leroy, 2005 ; Staszak, 2008 ; Roux, 20 I0).

L'objet de ce dossier est alors de permettre à des chercheurs de différentes disciplines de questionner la plage selon des éclairages qui ont été, jusqu'à récemment, souvent mis en veilleuse. Il ne suffit plus seulement d'affirmer que la plage est une construction sociale, mais de montrer comment ce lieu peut constituer un laboratoire pour travailler certains objets, au premier chef desquels se trouve le corps.

\section{De l'avènement À L’ÉVÉNEMENT : LA PLAGE, UN OBJET PROBLÉMATIQUE POUR LES SCIENCES SOCIALES}

\section{L'invention de la plage et après ?}

Si nous pouvons parler de la plage aujourd'hui en tant qu'objet des sciences sociales, c'est sans doute parce que le travail des sciences naturelles a permis préalablement son objectivation, a rendu possible la désactivation d'un certain imaginaire fantastique associé à l'espace marin. Mais par un parcours un peu compliqué, nous devons aussi au nouveau paradigme du "tournant culturel" d'avoir justement mis au jour le rôle des scientifiques (médecins, naturalistes, etc.) dans la "domestication" du rivage en Occident. En parlant de "territoire du vide", Alain Corbin (1988) a ainsi permis plus globalement de replacer la plage dans une histoire culturelle à travers laquelle s'est établi un retournement du regard. Nous savons désormais aussi le rôle décisif qu'a eu l'élite rentière dans la métamorphose de cet espace peu à peu approprié par les pratiques de loisir. L'artialisation dont a parlé Alain Roger (1997), ce procès d'esthétisation de certains éléments environnementaux par l'art pictural, en particulier, a permis d'inverser le signe négatif associé au rivage.

Mais si la plage est devenue un "paysage", les peintures du milieu du XVII siècle en ont aussi fait la scène du spectacle social (Knafou, 2000), le lieu inédit de l'exposition en public pour les classes dirigeantes à la recherche de nouvelles formes de distinction. Une nouvelle économie des apparences naît alors, qui connaîtra une longue carrière. Les implications engendrées par la mise au jour d'une telle matrice n'ont pas été suffisamment travaillées. Or la plage peut être appréhendée comme un espace public, lieu "où tous ceux qui s'y trouvent peuvent et doivent penser que tous les autres membres de la société pourraient l'y côtoyer" (Lévy, 1999), "espace sensible, dans lequel évoluent des corps, perceptibles et observables [...]" (Joseph, 1998). Cette qualité d'espace public propulse dans un même mouvement la plage du côté de l'urbanité, propriété qui a très peu retenu l'attention des sciences sociales jusqu'à présent, à l'exception de rares travaux (Coëffé, 2003 ; 2010a ; 2010b ; Pradel et Simon, 20।2), parmi lesquels figurent, dans le présent dossier, l'article de Thérèse Rouleau-Racco, ainsi que celui de Benjamin Pradel et Gwendal Simon. Si la plage est le lieu privilégié d'une mise en scène de l'ensauvagement et de l'état de nature, les pratiques qu'elles accueillent sont celles d'individus et de groupes qui y déplacent des valeurs et des normes urbaines, opérant un "transfert d'urbanité" (Coëffé, 2003) qui peut se lire aussi dans les fortes densités qui transforment régulièrement ce lieu, malgré le fantasme de la vacuité.

Alors que la plage manifeste en certains cas, ou plutôt en certaines circonstances de lieu, la convergence des multitudes, parmi lesquelles les touristes figurent en bonne place, quelles sont les questions que peuvent 
poser les sciences sociales sur le sens d'un objet raillé par les beaux esprits qui y voient d'abord un lieu de perdition, de retour à l'animalité, voire d'anomie sociale?

\section{De la difficulté de donner du corps à la plage : retour sur une journée d'étude}

Cette dernière interrogation ellemême ne va pas de soi, surtout lorsqu'elle suscite des explorations heuristiques qui peuvent déranger et qui sont donc encore timidement investies.

Si la plage fait l'objet de travaux dans les différentes sciences sociales (Urbain, 1995; Kaufmann, 2000 ; Johnston, 2005 ; Metusela et Waitt, 20I2), le corps, nous l'avons dit, reste encombrant dans le procès de compréhension de ce qui se joue par et à travers ce lieu. Ce fut pourtant notre pari d'organiser, en mars 2012, une journée d'étude à l'université d'Angers cherchant à interroger notamment les liens entre le corps et la plage ; cet événement nous a incités à proposer ce dossier à la revue Mondes du tourisme. Cinq communications avaient été présentées lors de cette journée ; une seule d'entre elles figure au sein de ce dossier, ce qui explique que des questions et des thématiques relatives à la plage aient - on le regrette - disparu : genre, sexualité, découverte de plages éloignées du foyer européen ${ }^{(1)}$.

Alors que le lieu touristique est régulièrement rattrapé par la norme scientifique qui s'autorise le jugement de valeur, ici plus facilement que partout ailleurs, la plage offre une caricature de cette posture : l'être humain y est régulièrement dépossédé de son humanité comme semblent l'évoquer sa posture horizontale et sa nudité, signes supposés d'un rebroussement vers l'animalité (Urbain, 1995 ; Coëffé, 20 l0b). Sans que cela ne provoque en effet le scandale, le "plageur", pour reprendre le mot de Jean-Didier Urbain (Urbain, 1995 ), s'apparente à l'ultime figure de "l'idiot culturel", dont le stigmate est porté à son apogée par le bronzage, pratique éminemment culturelle justement, dont l'invention a été travaillée par les historiens, les philosophes, les anthropologues et les sociologues (Andrieu, 2008 ; Ory, 2008), mais aussi, plus récemment, par les géographes (Équipe Mit, 2002, 2011 ; Coëffé, 2003 ; Coëffé, Guibert et Taunay, 2012).

Une des manières de transformer l'évidence en problème, de bousculer le statut de l'objet plage, est peutêtre de poser cette question naïve : pourquoi et comment des individus aux provenances et aux trajectoires différenciées parviennent-ils ici à tenir ensemble, dans la coprésence ? L'hypothèse de la coprésence présente l'avantage de traduire l'idée selon laquelle chaque individu habite la plage en étant présent à lui-même et aux autres. Nous n'avons plus affaire à l'abstraction statistique mais à un individu pris "dans la chair du monde" (Merleau-Ponty, 1945). Nous devons à Merleau-Ponty notamment d'avoir fait passer le corps du statut d'entrave, comme nous l'avait légué l'héritage plato- nicien et chrétien, à celui d'élément constitutif de notre existence, de l'habiter, au sens où la spatialité suppose une "interactivité" entre les acteurs et l'espace dans lequel ils évoluent (Lévy et Lussault, 2003).

C'est par et à travers son corps que l'individu habite, produit l'espace (Lefebvre, 2000), et la plage pourrait bien constituer un lieu rendant particulièrement visible cette proposition. Mais sur la plage ou ailleurs, nous pourrions dire aussi que deux corps, dans la mesure où chacun d'entre eux se déploie selon une certaine étendue, ne peuvent occuper le même site en même temps, le même topos (Lussault, 2009). Toutefois, ce constat fonctionne imparfaitement à partir du moment où nous observons des configurations à travers lesquelles les corps peuvent se superposer, s'enchevêtrer, comme c'est le cas de certaines actions liées aux sexualités ou aux violences (Lussault, 2007). Cela oblige donc à nous intéresser au réglage de la distance, problème géographique s'il en est. Quel sens prend la distance lorsqu'il n'est plus possible de déplacer sa serviette à moins d'empiéter sur celle des autres? En parlant de “proxémie”, Edward T. Hall (2000) s'est intéressé à la "perception et l'usage de l'espace par l'homme" jusqu'à définir notamment une "distance intime”, un écart en-deçà duquel la présence de l'autre peut être vécue comme intrusive; Erving Goffman (1973) évoque quant à lui la notion proche d' "offense territoriale”. Or cette distance varie selon les groupes mais aussi selon les indi- 
vidus, ce dont Edward T. Hall ne parle pas. Mais dans tous les cas, la gestion de la distance est travaillée par l'incorporation de normes culturelles.

Cela dit, le corps ne peut être réduit à une simple étendue, comme nous l'avons suggéré. Le corps humain n'est pas une chose comme les autres. Le corps est "le lieu où naissent et se manifestent nos désirs, nos sensations, nos émotions ; il est le moyen par lequel nous pouvons démontrer quelle sorte d'êtres moraux nous sommes [...]. Chaque personne entretient avec son corps une relation à la fois instrumentale et constitutive. Nous vivons une tension continuelle par rapport à notre existence physique : nous sommes complètement liés à notre corps tout en étant loin de lui. Contrairement aux autres corps $d u$ monde, mon corps semble donc 'adhérer' à moi au point que je ne peux m'en retirer. Je suis dans mon corps et les figures $d u$ retrait par rapport à cette insertion ont toujours quelque chose de métaphorique [...]. Au point que si l'on peut ignorer le corps, on ne peut pour autant l'annuler. Le corps est une sorte d'institution symbolique' qui lie l'objectivité du corps physique à la subjectivité du corps propre" (Marzano, 20 I0, p. 50-5I). Or, ce lien étant enveloppé par les normes collectives qui font du corps un enjeu de l'appartenance sociale (Bourdieu, 1977).

Si la plage est régulièrement représentée comme le lieu des possibles, lieu du hors-quotidien qui autorise le relâchement du self control, lieu de la recréation par excellence, qu'en est-il du régime de conventions qui s'y déploie, de la "civilisation des moeurs”, au sens de Norbert Elias (1973) ? Comment les individus coprésents font avec les règles sociales, notamment celles qui régulent les apparences et les gestes corporels? Quels sont les enjeux sociaux voire sociétaux que la plage permet d'observer ? La plage pourrait-elle être appréhendée de ce point de vue comme un lieu autonome, produit par et produisant ses propres logiques sociales, au sens où les conduites qui s'y déploient ne pourraient pas avoir lieu ailleurs comme Christophe Granger en fait l'hypothèse dans ce dossier ? N'y a-t-il pas au fond des cultures de la plage? En quoi et dans quelle mesure la plage ou plutôt les plages sont-elles des "espaces sexués et 'genrés" (Jaurand et Luze, 2004) ?

\section{LA PLAGE : \\ CONSTRUCTION D'UN TERRITOIRE}

\section{Désigner la plage :}

\section{territoire décor ou des corps ?}

L'utilisation du terme de territoire pour désigner les plages fréquentées par différents groupes sociaux et culturels ne correspond évidemment pas à l'acception traditionnelle de celui-ci, d'espace contrôlé et délimité, lié à un maillage administratif. D'ailleurs, Christophe Guibert montre que les limites spatiales de la plage peuvent être posées d'abord comme un problème avant que d'être imposées comme une évidence par le recours commode au découpage géomorphologique.

En revanche, la plage s'accorde bien avec une définition large du territoire comme celle proposée par Bernard Debarbieux (2003, p. 910) : "un agencement de ressources matérielles et symboliques capable de structurer les conditions pratiques de l'existence d'un individu ou d'un collectif social et d'informer en retour cet individu et ce collectif sur sa propre identité".

Avec la plage s'opère le double mouvement de territorialisation évoqué par Guy Di Méo (1998). On a d'une part une territorialisation descendante ou d'en haut, liée à des acteurs institutionnels. Puisqu'elles sont chargées de la gestion des plages, les municipalités règlementent les pratiques susceptibles de s'y déployer : prise d'arrêtés sur les interdictions et obligations relatives à l'usage de la plage, apposition de panneaux de réglementation et d'information qui sont la traduction dans l'espace d'un pouvoir réglementaire, division territoriale de la plage selon les activités - nautisme, sports de glisse, baignade. D'autre part et symétriquement, existe une territorialisation ascendante, qui pour Guy Di Méo "résulte des pratiques individuelles, des cheminements et des itinéraires personnels, de ces fourmillements $d u$ quotidien qui produisent des bassins de vie et d'action, mais aussi $d u$ lien collectif transitant par l'expérience et les rencontres de chacun" (2004, p. 20 I). La territorialisation de la plage passe par la présence régulière des individus, leurs 
sentiments, gestes, discours, actions de transformation du lieu et représentations de celui-ci. Il peut évidemment exister des conflits ou des transgressions entre les pratiques observées et les règles imposées par les municipalités.

Ces pratiques spatiales sont par définition incorporées. Paradoxalement, les recherches sur le tourisme ont longtemps négligé la dimension corporelle, au profit d'une approche désincarnée et comptable traduite par l'attention toute particulière portée à des flux touristiques indifférenciés (Deprest, 1997). Pourtant, nombre de pratiques touristiques reposent sur des performances corporelles (se baigner, s'allonger, escalader, etc.) qui permettent à l'individu d'incorporer l'espace. Le corps est en effet, selon Nancy Duncan, un médium qui assure la relation entre l'individu, d'une part, l'espace et les autres, d'autre part (1996). Il détermine ainsi l'expérience géographique de chacun, à travers l'orientation dans l'espace ou la perception de celuici par l'intermédiaire des cinq sens (Rodaway, 1994). L'apparence et la posture du corps sont révélateurs des manières de vivre d'un individu et de sa façon de se placer dans le jeu social (Ory, 2008). Il est aussi reconnu que l'expérience touristique est largement liée aux sensations éprouvées dans et par son corps (Urry, 1999). D’où la nécessité de mettre les pratiques et les "manières de se tenir" au cœur des études touristiques, en particulier sur le littoral (Duhamel et Violier, 2009).

\section{Plages, corps et territoires}

Le fonctionnement des plages repose largement sur un certain nombre de performances corporelles, communes aux individus qui les fréquentent, prenant leur sens dans un contexte social et spatial et contribuant à instituer ceux-ci en un collectif. Il s'agit de se déplacer, de repérer le lieu approprié aux pratiques du moment, mais aussi de s'approprier l'espace jusqu'à le privatiser à l'aide d'objets divers fonctionnant comme autant de marqueurs territoriaux (Urbain, 1995). Il s'agit aussi de trouver la bonne posture et d'effectuer les gestes qui sont socialement et culturellement attendus dans le contexte de normes qui informent et sont informées par les mondes de la plage. Ici s'opère en fait un sévère contrôle des apparences des corps, dont la subtile diversité renvoie à des critères d'âge ou d'identité sexuée et sexuelle, à côté de critères plus classiques de niveau social tôt repérés (Champagne, 1975 ; Chevallier, 2009). Comme le montre Christophe Granger dans ce dossier, l'histoire de la construction des plages comme territoires est largement l'histoire d'une police des corps, dans leur dimension sexuée en particulier. Une exposition au musée de Normandie (Caen) en 2009, consacrée à l'histoire régionale du tourisme, montrait une affiche de la "police des bains de mer" à Granville en 1837. L'article 1 était consacré au partage de la plage en trois secteurs : "une partie exclusivement réservée au bain des fermmes, une partie exclusivement réservée au bain des hommes habillés, une partie exclusivement réservée au bain des hommes non habillés". L'article 3 stipulait : "Il est interdit aux hommes de se promener ou de stationner sur ou le long de la grève occupée par les femmes durant le bain de celles-ci".

De telles injonctions et interdictions relatives aux apparences de la part de la puissance publique érigent la plage en territoire(s) des corps et assurent autant un contrôle des individus que l'appropriation d'un espace. Aujourd'hui, après deux siècles d'appropriation progressive d'un espace, à travers l'apprentissage de pratiques corporelles (tenir dans l'eau en équilibre, nager, glisser sur la surface des flots, se dorer la peau sans brûler, etc.), on aurait tort de croire à la disparition pure et simple des normes corporelles en Occident. On a plutôt assisté à leur déplacement au terme de conflits étalés sur des générations (Granger, 2009), avec peut-être une tendance contemporaine à un backlash (retour de bâton) évoqué aux États-Unis (Lagrange, 2010). D'abord, le tabou de la nudité est maintenu sur presque toutes les plages d'Europe, sauf par dérogation ou transgression (Jaurand, 2008) ; les seins nus sont le plus souvent ${ }^{(2)}$ interdits aux États-Unis, de même que sur la plage artificielle de Paris Plages, comme le rappellent Gwendal Simon et Benjamin Pradel dans ce dossier. Dans un pays du Sud largement ouvert au tourisme international comme la Turquie, les plages sont chaque année le théâtre de conflits qui tournent principalement autour 
du contrôle du corps féminin : la presse y relate chaque été des incidents nés de la présence sur une même plage de femmes turques intégralement voilées et d'autres en bikini, à l'origine d'une coexistence pas toujours pacifique.

Il existe cependant des cas, exceptionnels, de cohabitation de publics aux postures corporelles et aux tenues vestimentaires très différentes (Jaurand, 20 I0). Á la Grande Canarie, entre les stations de Maspalomas et de Playa del Inglès, la plage qui borde les célèbres dunes de Maspalomas propose un secteur de près de deux kilomètres de long dévolu à la pratique nudiste. On y remarque la cohabitation, dans une apparente indifférence, de plageurs pour la plupart nus et de promeneurs vêtus qui effectuent à pied le trajet entre les deux stations. Il existe ainsi des circulations de corps qui se croisent perpendiculairement : celle des passants, en file indienne assez serrée et parallèlement au rivage, et celle orthogonale à ce dernier, des nudistes qui se déplacent entre la zone de baignade et leur place sur le sable. Le territoire que constitue la plage résulte très clairement ici de l'entrecroisement de plusieurs territorialités, liées à des catégories d'acteurs aux perceptions de l'espace et aux pratiques différentes. Cet exemple assez rare de mélange d'apparences et de postures corporelles différentes montre aussi sans doute l'aboutissement d'un processus de dépénalisation de la nudité sur les plages dans l'Espagne post-franquiste (abandon de la légis- lation sur l'interdiction de la nudité sur les plages en 1989), d'une confrontation habituelle dans cette station de touristes venus de toute l'Europe et, plus largement, d'un long processus d'appropriation de la plage et de confrontation à l'altérité par les touristes européens. Tous ces traits font de la plage de Playa del Inglès un cas emblématique d'une certaine postmodernité. Celleci se marque aussi par l'hybridation des pratiques et des normes de présentation des corps dans des pays dits "du Sud" où le tourisme balnéaire national se développe.

\section{Et AILLEURS, \\ LA PLAGE PREND CORPS}

\section{Comment faire avec la plage hors de son foyer d'invention ?}

La plage comme paysage et scène sociale apparaît, ainsi que nous l'avons suggéré plus haut, comme une invention occidentale. Cela dit, cet "objet" ne peut être circonscrit à une "aire culturelle". Dans le contexte d'une mondialisation ${ }^{(3)} \mathrm{du}$ tourisme qui s'accélère, de nouveaux espaces littoraux émergent en tant que plages fréquentées par des sociétés n'appartenant pas au foyer où est né le phénomène touristique. En Chine, par exemple, des lieux tels que l'île de Hainan accueillent chaque année près de 20 millions de touristes, quasiment tous résidants chinois dans le pays ${ }^{(4)}$. Cette situation, toutes proportions gardées, n'est pas spécifique à la Chine mais se constate dans différents pays encore qualifiés “d'émer- gents" et qui pourtant abritent un tourisme intérieur important (Cabasset, Peyvel, Sacareau et Taunay, 2010).

Ainsi, au moment où de nouveaux territoires sont incorporés par le phénomène touristique, comment comprendre les ressorts de pratiques de plages qui ne sont pas en filiation directe avec celles du foyer européen où est apparu le tourisme près de deux cents ans plus tôt, mais qui révèlent plutôt des discontinuités spatiales, temporelles et culturelles ? C'est là une interrogation qui ne figure pas dans ce dossier - on le déplore sans pouvoir y remédier - tant les recherches disponibles ou en cours sont en nombre limité. Au sein de la dernière livraison d'importance en langue française sur le thème de la plage, le numéro 69 de la revue Géographie et cultures, seul un article traitait d'une plage aux antipodes de l'Europe (Lageiste et Rieucau, 2009). Au travers de l'exemple de Mui Ne (Viêt Nam), Emmanuelle Peyvel (dont le texte a été retravaillé plus tard dans sa thèse) montrait la "juxtaposition spatiale" de deux espaces fondés sur des pratiques, des représentations et donc des normes presque opposées. Si bien que ce que valorisent les touristes occidentaux dans et à travers un espace devient répulsif pour les touristes vietnamiens, et inversement (Peyvel, 2009, p. 279). Les logiques des territoires touristiques de plage sont ici informées par l'opposition de normes spatiales, la pudeur et la blancheur 
dominant les unes quand les autres se fondent sur le dévoilement des corps et la recherche du bronzage.

Si le rapprochement de pratiques touristiques clairement différenciées peut avoir une fonction heuristique, la comparaison internationale, même in situ, peut aussi présenter des limites si elle est confondue avec la fusion. La compréhension de la mondialisation touristique, sur les plages en particulier, ne consiste en effet pas seulement à analyser comment des territoires sont incorporés à celleci, mais peut également montrer comment ces territoires nous informent sur les processus d'émergence de nouveaux foyers touristiques au sein desquels, on en fait l'hypothèse, s'inventent peut-être de nouvelles manières de faire avec l'espace des plages. Si la circulation des modèles touristiques s'est d'abord faite à partir de l'Europe, considérée comme un centre, les nouvelles "frontières $d u$ tourisme" (Peyvel, Sacareau et Taunay, 2015) permettent de renverser le regard et d'appréhender les nouvelles circulations de pratiques, voire de modèles. De nouveaux foyers de diffusion du tourisme se font jour. Il ne s'agit plus de ces anciens espaces colonisés, périphéries du tourisme international occidental jusqu'au début des années 1990 (Cazes, 1990), mais bien de lieux où se joue l'expression originale d'un rapport urbain à la plage.

\section{Des rapports au corps à explorer}

Ce champ de recherche reste à explorer dans le cas de ces plages méconnues où les corps peuvent être découverts alors que l'acte de dévoilement peut poser problème pour une majorité de "plageurs". Dans le cas de la Chine, cela pose ainsi l'hypothèse d'une éventuelle appropriation du hâle, alors que la peau blanche est érigée en canon de beauté dans le pays, la fadeur étant reconnue comme une qualité sociétale qui pourrait également concerner la couleur de la peau (Jullien, 2005). Certains individus chinois, une minorité à ce jour, s'autorisent toutefois à mettre à l'épreuve cette esthétique et se mettent à bronzer, à l'instar des surfeurs chinois de l'île de Hainan (Guibert et Taunay, 20/3), ce qui peut s'expliquer, en partie, par les trajectoires sociales de ces derniers : le bronzage deviendrait une ressource économique, voire symbolique, pour des individus socialisés, par ailleurs faiblement dotés en capital culturel (Coëffé, Guibert et Taunay, 2014).

Mais ailleurs, comment interpréter les rapports au corps qui se jouent sur les plages ? Dans les rares travaux sur la construction sociale et culturelle de la couleur de la peau dans les sociétés d'Asie, la valeur positive de la peau blanche serait ici un invariant anthropologique ( $L$, Min, Belk, Kimura et Bahl, 2008). Or cette posture ne permet pas de penser les mutations en cours concernant les codes corporels de beauté, comme le montrent les travaux empiriques précités. À Singapour, par exemple, il existe "plusieurs attitudes envers la couleur de la peau aujourd'bui [...]. D'un côté les lotions corporelles, les masques pour le visage qui promettent une 'peau de porcelaine'. [...] Plus récemment, cependant, des crèmes et des huiles pour favoriser le bronzage sont devenues de plus en plus populaires" (Winter, Theo et Chang, 2009, p. 3). On pourra alors objecter que Singapour est un des hauts lieux de la mondialisation contemporaine, que cette ville, comme Shanghai et ses instituts de bronzage, est au cœur des faisceaux de pratiques mondialisées et que le bronzage ne serait alors qu'un avatar du bronzage né en Occident. C'est en ce sens que le sinologue René Barbier (2002) note qu'en Chine "le rapport au corps s'infléchit en copiant le modèle occidental". Le bronzage en Chine n'est-il alors que l'ultime étape de sa circulation à travers le monde, entamée à Hawaï il y a un peu plus de cent ans ? Cette pratique corporelle va-t-elle devenir la norme sur les plages chinoises, ou bien est-ce là un phénomène éphémère, limité socialement à certaines classes aisées, minoritaire à terme par rapport à la manifestation croissante en Europe du whitening (l'application de crèmes visant à blanchir la peau), conséquence d'une circulation d'un modèle esthétique venu de Corée du Sud ? Ou ce dernier modèle s'amplifiera-t-il pour devenir le référent principal avec la massification du tourisme international chinois dans le monde?

Dans ce dossier, nous invitons le lecteur à considérer la plage comme un objet scientifique paradoxal : non comme un territoire de la vacuité, mais comme un territoire 
chargé de signes; non comme une anomie sociale, mais comme un révélateur des contradictions, conflits et dynamiques qui traversent les corps, individuels et sociaux ; non comme le lieu d'un spectacle futile, mais comme une scène sociale où se jouent des représentations dont les scripts résultent d'un long travail d'élaboration, mené et enrichi sur plusieurs générations. Bref, nous sommes conscients de la richesse de cet objet qui ne se laisse pas facilement saisir ou enfermer. Nous invitons alors le lecteur à entrer dans le décor et à circuler dans ce territoire des corps qui traverse le temps et les espaces.

\section{Notes}

( I) Le présent dossier n'est donc pas une simple compilation d'actes de cette journée du 20 mars 2012. Les cinq communications présentées étaient: Philippe DuHAMEL, “"Et tous ces corps étendus sur la plage' : une contre-évolution de l'humanité ?" ; Christophe GrANGER, "Une journée à la plage ou la difficile pacification d'un territoire (1920-1940)"; Francine BARTHE, "Le Brésil et la plage : constructions/déconstructions du stéréotype de l'identité brésilienne à travers l'exemple de Salvador de Bahia" ; Emmanuel JaURAND, "Gay heaven on the beach. Corps, homosexualité et territorialités" ; Yves RAIBAUD, "Des lieux qui assouplissent la norme : la plage du Porge (Gironde)".

(2) II existe toutefois des exceptions, comme à New-York où la pratique des seins nus est autorisée depuis 1992.

(3) Par mondialisation, on entend la mise en relation des lieux à l'échelle de la planète, à travers la circulation d'objets (idéels et matériels) et leur réception par les individus et groupes sociaux localisés (voir Retaillé, 2007).

(4) Les recherches sur le terrain évoquées dans ce chapitre (Benjamin Taunay) ont été en partie financées par le projet I IHYJDYY06 (Centre de recherches provincial de la culture et de l'économie maritimes du Zhejiang).

\section{Références bibliographiques}

Bernard ANDRIEU, Bronzage. Une petite histoire du soleil et de la peau, CNRS éditions, 2008.

Jean-René BARBIER, "L'imaginaire du corps dans la Chine contemporaine : une approche transversale", Notes pour le séminaire de recherche en DEA de décembre 2002

[http://www.barbier-rd.nom.fr/ChineCorpslmaginaire.html].

Pierre BOURDIEU, "Remarques provisoires sur la perception du corps", Actes de la recherche en sciences sociales, n 14, 1977.

Christine Cabasset, Emmanuelle Peyvel, Isabelle Sacareau

et Benjamin TAUNAY, "De la visibilité à la lisibilité du

tourisme domestique en Asie : quelques réflexions à partir des cas chinois, indiens, indonésiens et vietnamiens", Espace Populations Sociétés, $n^{\circ}$ 2-3, 2010.

Georges CAZES, Les nouvelles colonies de vacances? Le tourisme international à la conquête du Tiers-monde, tome I, coll. "Tourismes et Sociétés", L'Harmattan, 1990.

Patrick Champagne, "Les paysans à la plage", Actes de la recherche en sciences sociales, $n^{\circ} 2,1975$.

Dominique Chevaluier, "Un territoire de cospatialités en recomposition : la plage de Maguelonne", Géographie et cultures, $n^{\circ}$ 68, 2009.

Vincent CoËFfé, Touristicité idéale. Hawaii, un parcours utopique, thèse de doctorat en géographie, Université de Rouen, 2003.

Vincent CoËFfÉ, "Le tourisme, fabrique d'urbanité. Matériaux pour une théorie de l'urbain", Mondes du tourisme, $n^{\circ} 2$,

2010 a.

Vincent CoëFFÉ, “La plage, fabrique d'une touristi(cité) idéale", L'Information géographique, vol. 74, n 3, $2010 \mathrm{~b}$.

Vincent COËFFÉ, Christophe GUIBERT et Benjamin TAUNAY, "Émergences et diffusions mondiales du surf. De l'invention à la mise à l'épreuve de normes corporelles", Géographie et cultures, $n^{\circ} 82,2012$.

Vincent Coëffé, Christophe GUIBERT et Benjamin TAUNAY, 
"L'aire du bronze : jalons pour une analyse de la circulation et de l'appropriation du hâle (de Hawaï à Hainan)",

L'Information géographique, vol. 78, n I, 2014.

Alain CORBIN, Le territoire du vide. L'Occident et le désir du rivage 1750-1840, Flammarion, 1988

Bernard DeBARBIEUX, entrée "Territoire", dans Jacques Lévy et Michel Lussault (dir.), Dictionnaire de la géographie et de l'espace des sociétés, Belin, 2003.

Florence DePREST, Enquête sur le tourisme de masse. L'écologie face au territoire, coll. "Mappemonde", Belin, 1997.

Guy Dı MÉo, Géographie sociale et territoires, Nathan, 1998. Guy Dı Méo, "Une géographie sociale dans le triangle des rapports hommes, sociétés, espaces", Bulletin de l'Association de géographes français, vol. 81, $n^{\circ} 2,2004$.

Philippe Duhamel et Philippe VIOLIer, Tourisme et littoral : un enjeu du monde, Belin, 2009.

Nancy DunCAN (dir.), Bodyspace. Destabilizing geographies of gender and sexuality, Routledge, 1996.

Norbert ELIAS, La civilisation des mœurs, Calmann-Lévy, 1973.

Équipe Mit, Tourismes I. Lieux communs, Belin, 2002.

Équipe Mit, Tourismes 3. La révolution durable, Belin, 201 I.

Erving GoffMAN, La mise en scène de la vie quotidienne. 2. Les relations en public, coll. "Le sens commun", Éditions de Minuit, 1973.

Christophe Granger, Les corps d'été, éditions Autrement, 2009.

Christophe GUiBerT et Benjamin TAUnAY, "From political pressure to cultural constraints: The prime dissemination of surfing in Hainan", The Journal of China Tourism Research, vol. $9, n^{\circ} 3,2013$

Edward T. Hall, La Dimension cachée, coll. "Essais", Éditions du Seuil, 1984 (I 959 pour la Ire édition).

Edward T. HaLl, "Proxémique", dans Yves Winkin (dir.), La nouvelle communication, Éditions du Seuil, 2000.

Emmanuel JAURAND, "Les plages nudistes, une exception occidentale ?", Géographie et cultures, n 67, 2008.

Emmanuel JAURAND, Construire des territoires d'un autre genre? Perspectives de recherche sur des territorialités marginales dans l'espace touristique, dossier inédit d'HDR préparé sous la direction du professeur J.-C. Gay et soutenu à l'université de NiceSophia Antipolis le 19 novembre 2010, vol. 3.

Emmanuel JAURAND et Hubert DE LUZE, "Ces plages où les genres s'affichent? Les territoires du nu sur la Côte d'Azur", dans Christine Bard (dir.), Le genre des territoires. Féminin, masculin, neutre, Presses universitaires d'Angers, 2004.

Lynda JOHNSTON, "Transformative tans? Gendered and raced bodies on beaches", The New Zealand Geographer, 61, 2005. Isaac JOSEPH, La Ville sans qualité, Éditions de l'Aube, 1998. François JuLlien, Le Nu impossible, coll. "Points Essais", Le Seuil, 2005.

Jean-Claude Kaufmann, Corps de femmes, regards d'hommes. Sociologie des seins nus, Nathan, 2000.

Rémy KNAFOU, "Scènes de plage dans la peinture hollandaise du XVIl siècle : l'entrée de la plage dans l'espace des citadins", Mappemonde, $n^{\circ}$ 58, 2000.

Jérôme LAGEISTE et Jean RIEUCAU (dir.), "La plage : un territoire atypique", Géographie et cultures, n 67, 2009.

Hugues LAgrange, Le déni des cultures, Seuil, 2010.

Henri LefeBVRe, La production de l'espace, Anthropos, 2000

(4e édition).

Stéphane LEROY, “Le Paris gay. Éléments pour une géographie de l'homosexualité", Annales de géographie, n 646, 2005.

Jacques LÉVY, Le toumant géographique. Penser l'espace pour lire le monde, coll. "Mappemonde", Belin, 1999.

Jacques LÉvY et Michel LusSAULT, entrée "Habiter", dans Jacques Lévy et Michel Lussault (dir.), Dictionnaire de la géographie et de l'espace des sociétés, Belin, 2003.

Eric P. H. LI, Hyun Jeong MIN, Russel W. BelK, Junko

KIMURA et Shalini BAHL, "Skin Lightening and Beauty in Four 
Asian Cultures", Advances in Consumer Research, n 35, 2008.

Michel LusSAULt, L'Homme spatial. La construction sociale de l'espace humain, coll. "La couleur des idées", Éditions du Seuil, 2007.

Michel Lussault, De la lutte des classes à la lutte des places, Grasset, 2009.

Michela Marzano, La philosophie du corps, coll. "Que saisje?", Puf, 2010 (2édition).

Maurice Merleau-PonTY, Phénoménologie de la perception, coll. "Tel", Gallimard, 1945.

Christine MetuseLA et Gordon WAITt, Tourism and Australian Beach Cultures. Revealing Bodies, Channel View Publications, 2012.

Pascal ORY, L'invention du bronzage, éditions Complexe, 2008. Roland PASKOFF, Les plages vont-elles disparaître?, Le

Pommier, 2005.

Emmanuelle PEYVEL, L'émergence du tourisme domestique au Viet-Nam : lieux, pratiques et imaginaires, Thèse de doctorat en géographie, Université de Nice-Sophia Antipolis, 2009. Emmanuelle PEYVEL, Isabelle SACAREAU et Benjamin TAUNAY, Les nouvelles frontières du tourisme, l'accès à un tourisme mondialisé, Presses universitaires de Rennes, 2015 (à paraître).

Benjamin Pradel et Gwendal Simon, "Quand le citadin jour au plagiste. Paris Plages, des référents touristiques dans le quotidien urbain", Espaces et Sociétés, n 151, 2012.

Denis RetallLé (dir.), La mondialisation, Nathan, 2007.

Paul RoDAWAY, Sensuous geographies. Body, sense and place,

Routledge, 1994.

Alain ROGER, Court traité du paysage, Gallimard, 1997.

Jean-François STASZAK, "Danse exotique, danse érotique.

Perspectives géographiques sur la mise en scène du corps de l'Autre (XVIII'-XX|' siècles)", Annales de géographie, n 660-66I, 2008.

Mathis StOck et Anne VolveY, entrée "Sexualité", dans Jacques Lévy et Michel Lussault (dir.), Dictionnaire de la géo- graphie et de l'espace des sociétés, Belin, 2003.

Jean-Didier URBAIN, Sur la plage. Mœurs et coutumes balnéaires (XIX - XXe siècles), coll. "Petite Bibliothèque Payot", Payot, 1995.

John URRY, The Tourist Gaze. Leisure and Travel in Contemporary Societies, Sage, 1999.

\section{Tim Winter, Peggy Teo et Tou Chuang Chang,}

"Rethinking tourism in Asia, in Asia on tour: exploring the rise of Asian tourism", dans Tim Winter, Peggy Teo et Tou Chuang Chang, Asia on Tour. Exploring the Rise of Asian Tourism, Routledge, 2009. 